\title{
ON THE UNIQUENESS OF SOLUTIONS TO HYPERBOLIC BOUNDARY VALUE PROBLEMS
}

\author{
BY
}

C. C. TRAVIS

ABSTRACT. The paper is concerned with the uniqueness of solutions to non-well-posed hyperbolic boundary value problems. Both regular and singular boundary value problems are considered. For the singular problem a class of boundary conditions is considered that has not appeared in the literature before in connection with this problem.

1. Introduction. It is well known that the Dirichlet problem for hyperbolic equations does not in general constitute a well-posed problem. In the case of the one-dimensional wave equation $u_{t t}-u_{x x}=0$, for example, Bourgin and Duffin [2] have shown that uniqueness to the Dirichlet problem for a rectangle with sides parallel to the coordinate axis holds if and only if the ratio of the sides of the rectangle is an irrational number. The Dirichlet problem for the one-dimensional wave equation has also been considered by Picone [9], John [8], and Fox and Pucci [7]. Dunninger and Zachmanoglou have extended the result to $n$-dimensional wave equations [5], and to more general hyperbolic equations in cylindrical domains [6]. Similar results for the Neumann problem for the $n$-dimensional wave equation have been obtained by Sigillito [10]. The result has been extended to ultrahyperbolic equations by Diaz and Young [3], and to singular hyperbolic equations by Young [11], and Dunninger and Weinacht [4]. Recently, Abdul-Latif and Diaz [1] have given a geometric proof of the original one-dimensional Bourgin and Duffin result under more general DirichletNeumann boundary conditions.

In all of the above papers, with the exception of Abdul-Latif and Diaz, the basic technique of proof is the same. An energy integral argument is used along with the fact that a certain class of eigenfunctions forms a complete set. We wish to present a proof which we believe is more direct, and at the same time demonstrates the close relationship between uniqueness results for hyperbolic boundary value problems and the Fredholm alternative. This method of

Presented to the Society, November 9, 1974; received by the editors November 26, 1974 and, in revised form, March 25, 1975.

AMS (MOS) subject classifications (1970). Primary 34B05, 35L20.

Key words and phrases. Non-well-posed, singular hyperbolic equation, limit point, limit circle. 
proof also allows for much more general boundary conditions than have been previously considered. However, the primary benefit of this new method of proof is that it yields new insight when applied to the class of singular hyperbolic boundary value problems considered by Young [11]. The major results in this paper will be for this class of singular boundary value problems, but first we will consider the regular case because of its simplicity.

2. The regular case. In order to better demonstrate the close relationship between the Fredholm alternative and the uniqueness of solutions to hyperbolic boundary value problems, we shall restrict our attention to the one-dimensional wave equation

$$
\begin{aligned}
u_{t t}-u_{x x} & =f(x, t) & & \text { in } R, \\
u & =0 & & \text { on } \partial R,
\end{aligned}
$$

where $R=\{(x, t): 0 \leqslant x \leqslant a, 0 \leqslant t \leqslant b\}$. We assume that $f \in C^{\prime}(R)$ and $f=0$ on $\partial R$.

In general, existence of solutions to the nonhomogeneous boundary value problem (2.1) is difficult to establish, and still sppears to be an open question for arbitrary $f$. However, since our main purpose in discussing problem (2.1) is to demonstrate its relationship with the Fredholm alternative, we shall circumvent the difficulties involved in establishing existence in the general case by assuming that

$$
\int_{0}^{a} f(x, t) \sin \frac{k \Pi}{a} x d x \equiv 0
$$

for all but a finite number of values of $k=1,2,3, \ldots$.

THEOREM 2.1. Problem (2.1) has a unique solution $u \in C^{2}(R)$ if and only if $a / b$ is an irrational number. If $a / b$ equals some rational number $m / n$, then (2.1) has a nonunique solution if

$$
\int_{0}^{a} \int_{0}^{b} \sin \frac{m \Pi x}{a} \sin \frac{n \Pi t}{b} f(x, t) d t d x=0 .
$$

Otherwise, problem (2.1) has no solution.

Proof. Define

$$
h_{k}(t)=\frac{2}{a} \int_{0}^{a} u(x, t) \sin \frac{k \Pi x}{a} d x, \quad f_{k}(t)=\frac{2}{a} \int_{0}^{a} f(x, t) \sin \frac{k \Pi x}{a} d x,
$$

for $k=1,2,3, \ldots$. Then $h_{k}(t)$ satisfies the inhomogeneous boundary value problem

$$
h_{k}^{\prime \prime}(t)+\frac{k^{2} \Pi^{2}}{a^{2}} h_{k}(t)=f_{k}(t), \quad h_{k}(0)=h_{k}(b)=0
$$


It follows from our assumptions on $f(x, t)$ that if $\left\{h_{k}(t)\right\}$ is a sequence of solutions to (2.2) then $u(x, t)=\Sigma_{k=1}^{\infty} h_{k}(t) \sin (k \Pi x / a)$ is a twice continuously differentiable solution of (2.1). Thus, it follows that the boundary value problem (2.1) has a unique solution if and only if the problem (2.2) has a unique solution for $k=1,2, \ldots$.

However, problem (2.2) has a unique solution if and only if $k^{2} \Pi^{2} / a^{2}$ is not equal to any of the eigenvalues of $h^{\prime \prime}(t)+\lambda h(t)=0, h(0)=h(b)=0$, that is, if and only if $k^{2} \Pi^{2} / a^{2} \neq n^{2} \Pi^{2} / b^{2}$, for $k=1,2, \ldots$, and $n=1,2, \ldots$.

Thus, we see that problem (2.1) has a unique solution if and only if $a / b$ is irrational. The remainder of the proof follows directly from the Fredholm alternative.

A similar result can be stated for the equation

$$
\begin{gathered}
u_{t t}-\sum_{i, j=1}^{n} D_{i}\left(a_{i j}(x) D_{j} u\right)+c(x) u=f(x, t), \\
u(x, 0)=u(x, a)=0 \text { for } x \in G, \quad \partial u / \partial v+\sigma(x) u=0 \text { on } \partial G \times[0, a] .
\end{gathered}
$$

However, we shall forego this extension and instead concentrate on an extension to a class of singular hyperbolic boundary value problems. First we need to establish some results about singular boundary value problems for ordinary differential equations.

3. Some preliminary results. Consider the homogeneous equation

$$
-\left(p(t) \phi^{\prime}\right)^{\prime}+q(t) \phi=\lambda s(t) \phi, \quad 0<t<a,
$$

where the point $a$ is a regular endpoint and the point zero is a singular endpoint, that is $p(0)=0$. A solution of (3.1) that satisfies

$$
\int_{0}^{a} s(t)|\phi(t)|^{2} d t<\infty,
$$

is said to have finite $S$ norm over $(0, a)$. The fundamental result concerning singular differential equations was obtained by $\mathrm{H}$. Weyl.

WEYL'S THEOREM. Let a be a regular point and 0 a singular point of equation (3.1).

(i) If for some particular value of $\lambda$ every solution of (3.1) is of finite $S$ norm over $(0, a)$, then for any other value of $\lambda$, every solution is again of finite $S$ norm over $(0, a)$.

(ii) For every $\lambda$ with $\operatorname{Im} \lambda \neq 0$, there exists at least one solution of finite $S$ norm over $(0, a)$.

We say that we have the limit circle case at 0 when all solutions are of finite $S$ norm over $(0, a)$. We have the limit point case at 0 when for some $\operatorname{Im} \lambda$ $\neq 0$ there is exactly one solution of finite $S$ norm over $(0, a)$. 
We shall now consider the equation

$$
\left(t^{k} \phi^{\prime}\right)^{\prime}+t^{k} \lambda \phi=0 \text { in } 0<t<a .
$$

The point $t=0$ is singular, and for $\lambda=0$ we find that $\phi_{1}(t)=1$ and $\phi_{2}(t)=$ $t^{1-k}$ are independent solutions of (3.3). Clearly, $\int_{0}^{a} t^{k}\left|\phi_{1}(t)\right|^{2} d t<\infty$ if and only if $-1<k$ and $\int_{0}^{a} t^{k}\left|\phi_{2}(t)\right|^{2} d t<\infty$ if and only if $k<3$. Thus, for equation (3.3) we have the limit circle case at $t=0$ if and only if $-1<k<3$; otherwise, we have the limit point case. We shall consider the limit point case first. If we impose the boundary condition $\phi(a)=0$ at the regular point $a$, and the boundary condition $\int_{0}^{a} t^{k}|\phi(t)|^{2} d t<\infty$ at the singular point, we are led to the singular eigenvalue problem

$$
\begin{gathered}
\left(t^{k} \phi^{\prime}\right)^{\prime}+t^{k} \lambda \phi=0, \quad 0<t<a, \\
\int_{b}^{a} t^{k}|\phi(t)|^{2} d t<\infty, \quad \phi(a)=0 .
\end{gathered}
$$

In general, a singular eigenvalue problem in the limit point case, like (3.4), can have both a continuous spectrum and a point spectrum, or it can have a continuous spectrum and no point spectrum, or it can have a pure point spectrum.

However, for equation (3.4) we have the following result.

THEOREM 3.1. For $k \notin(-1,3)$, the singular eigenvalue problem (3.4) has a pure point spectrum and the eigenfunctions form a complete orthonormal set with weight function $t^{k}$.

Proof. It is not difficult to verify that for $3 \leqslant k$

$$
G(t, \xi)= \begin{cases}\left(a^{1-k}-t^{1-k}\right) /(1-k) & \text { for } \xi<t, \\ \left(a^{1-k}-\xi^{1-k}\right) /(1-k) & \text { for } t<\xi ;\end{cases}
$$

and for $k \leqslant-1$

$$
G(t, \xi)= \begin{cases}\xi^{1-k}\left(a^{1-k}-t^{1-k}\right) /(1-k) a^{1-k} & \text { for } \xi<t, \\ t^{1-k}\left(a^{1-k}-\xi^{1-k}\right) /(1-k) a^{1-k} & \text { for } t<\xi ;\end{cases}
$$

are the Green's functions associated with the operator $L \phi=-\left(t^{k} \phi^{\prime}\right)^{\prime}, \int_{0}^{a} t^{k}|\phi(t)|^{2} d t$ $<\infty, \phi(a)=0$. The singular eigenvalue problem (3.4) can thus be translated into the integral equation

$$
\phi(t)=\lambda \int_{0}^{a} G(t, \xi) \xi^{k} \phi(\xi) d \xi
$$

or

$$
\mu \psi(t)=\int_{0}^{a} K(t, \xi) \psi(\xi) d \xi
$$


where $\mu=1 / \lambda, \psi(t)=t^{k / 2} \phi(t), K(t, \xi)=t^{k / 2} G(t, \xi) \xi^{k / 2}$. It is easily verified that $\int_{0}^{a} \int_{0}^{a}|K(t, \xi)|^{2} d t d \xi<\infty$. Thus $K(t, \xi)$ is a real symmetric Hilbert-Schmidt kernel, and it follows that the singular eigenvalue problem (3.4) has only point spectrum and that the eigenfunctions form a complete orthonormal set with weight $t^{k}$.

COROLlARY 3.2. If $f(t)$ has finite $t^{k}$ norm over $(0, a)$, where $k \notin(-1,3)$, then the Fredholm alternative holds for the singular equation

$$
\left(t^{k} \phi^{\prime}\right)^{\prime}+\lambda t^{k} \phi=t^{k} f(t), \quad \int_{0}^{a} t^{k}|\phi(t)|^{2} d t<\infty, \quad \phi(a)=0 .
$$

We shall now treat the limit circle case. If we define

$$
W(u, v ; t) \equiv u v^{\prime}=u^{\prime} v,
$$

then it is known that every solution in the limit circle case satisfies the condition $\lim _{t \rightarrow 0^{+}} t^{k} W(u, \bar{u} ; t)=0$. Thus, we are led to consider the problem

$$
\left(t^{k} \phi^{\prime}\right)^{\prime}+\lambda t^{k} \phi=0, \lim _{t \rightarrow 0^{+}} t^{k} W(\phi, \bar{\xi} ; t)=0, \phi(a)=0,
$$

where $\xi\left(t, \lambda_{0}\right)$ is a possibly complex-valued solution of $\left(t^{k} \xi^{\prime}\right)^{\prime}+\lambda_{0} t^{k} \xi=0$ satisfying $\lim _{t \rightarrow 0^{+}} t^{k} W(\xi, \bar{\xi} ; t)=0$. It is well known that the limit circle case with the above boundary conditions always leads to a pure point spectrum and that the eigenfunctions form a complete orthonormal set with weight $t^{k}$.

CoRollary 3.3. If $f(t)$ has finite $t^{k}$ norm over $(0, a)$, where $-1<k<3$, then the Fredholm alternative holds for the singular equation

$$
\left(t^{k} \phi^{\prime}\right)^{\prime}+\lambda t^{k} \phi=t^{k} f(t), \lim _{t \rightarrow 0^{+}} t^{k} W(\phi, \bar{\xi} ; t)=0, \quad \phi(a)=0 .
$$

4. The singular case. In this section we shall investigate the uniqueness of solutions to the singular hyperbolic boundary value problem

$$
\begin{gathered}
u_{t t}+\frac{k}{t} u_{t}-\sum_{i, j=1}^{n} D_{i}\left(a_{i j}(x) D_{j} u\right)+c(x) u=0 \\
u(x, 0)=u(x, a)=0 \quad \text { for } x \in G, \quad \partial u / \partial v+\sigma(x) u=0 \quad \text { on } \partial G \times[0, a],
\end{gathered}
$$

where $k$ is a real parameter, $-\infty<k<\infty$. We assume $x=\left(x_{1}, x_{2}, \ldots, x_{n}\right)$ represents a point in $E^{n}$ and $a_{i j}(x)$ is of class $C^{1}$ in a domain $D=\{(x, t): x \in G$; $0 \leqslant t \leqslant a\}$ where $G$ is a bounded regular domain in $E^{n}$. It is also assumed that $c(x)$ is continuous, $a_{i j}=a_{i j}$, and that the principal part of equation (3.1) is uniformly elliptic, i.e., $\Sigma a_{i j}(x) \xi_{i} \xi_{j} \geqslant \mu \mid \xi^{2} \quad(\mu>0)$ for all $n$-tuples $\xi=\left(\xi_{1}, \xi_{2}\right.$, $\left.\ldots, \xi_{n}\right)$. We shall denote by $\partial / \partial \nu$ the transverse derivative defined by 


$$
\frac{\partial}{\partial \nu}=\sum_{i, j=1}^{n} a_{i j}(x) \cos \left(\nu ; x_{i}\right) \frac{\partial}{\partial x_{j}}
$$

where $\nu$ is the exterior normal to $\partial G$.

Equation (4.1) is a generalization of the well-known Euler-Poisson-Darboux equation which has been the object of extensive research. E. C. Young [11] has studied boundary value problems for equation (4.1) under less general boundary conditions than considered here. He made special notice of the cases $k<0$ and $0 \leqslant k$. We shall demonstrate that the two important cases are $k \in(-1,3)$ and $k \notin(-1,3)$. It should be pointed out that the results obtained in this section are new even in the case $k=0$, where they extend [2], [6], [7] by considering more general boundary conditions.

THEOREM 4.1. If $k \notin(-1,3)$ and $u \in C^{2}(\bar{G} \times(0, a))$ is a solution of

$$
\begin{aligned}
u_{t t}+\frac{k}{t} u_{t}- & \sum_{i, j=1}^{n} D_{i}\left(a_{i j}(x) D_{j} u\right)+c(x) u=0, \\
& \partial u / \partial v+\sigma(x) u=0 \quad \text { on } \partial G \times(0, a), \\
& \int_{0}^{a} t^{k}|u(x, t)|^{2} d t<\infty, \quad u(x, a)=0 \quad \text { for } x \in G
\end{aligned}
$$

then $u \equiv 0$ if and only if

$$
J_{|1-k| / 2}\left(\sqrt{\lambda_{m}} a\right) \neq 0
$$

where $J_{p}(z)$ is the Bessel's function of the first kind of order $p$, and $\lambda_{m}$ are the positive eigenvalues of the elliptic eigenvalue problem

$$
\begin{aligned}
& -\sum_{i, j=1}^{n} D_{i}\left(a_{i j}(x) D_{j} \psi\right)+c(x) \psi=\lambda \psi \quad \text { in } G \\
& \partial \psi / \partial \nu+\sigma(x) \psi=0 \quad \text { on } \partial G
\end{aligned}
$$

Proof. Suppose there exists a positive eigenvalue $\lambda_{n}$ of the elliptic eigenvalue problem (4.4) such that $J_{|1-k| / 2}\left(\sqrt{\lambda_{n}} a\right)=0$. Let $\psi_{n}(x)$ be the normalized eigenfunction corresponding to $\lambda_{n}$. Then the function

$$
w(x, t)=t^{(1-k) / 2} J_{|1-k| / 2}\left(\sqrt{\lambda_{n}} t\right) \psi_{n}(x)
$$

is a nontrivial solution to the problem (4.2).

Conversely, suppose that condition (4.3) holds and that there exists a nontrivial solution $u(x, t)$ of the hyperbolic boundary value problem (4.2). Let $\left\{\psi_{i}(x)\right\}$ and $\left\{\phi_{j}(t)\right\}$ be the normalized eigenfunctions of problems (4.4) and (3.4), respectively. Then, the set $\left\{\psi_{i}(x) \phi_{j}(t)\right\}$ is a complete orthonormal set for the Hilbert space 


$$
H=\left\{f: G \times(0, a) \rightarrow R^{1} \mid \int_{0}^{a} \int_{G} t^{k} f^{2}(x, t) d x d t<\infty\right\}
$$

The continuity assumptions on $u$ and the fact that $u$ satisfies the integral boundary condition in (4.2) imply that $u \in H$. Thus, we can write

$$
u(x, t)=\sum_{i, j=1}^{\infty} \alpha_{i j} \psi_{i}(x) \phi_{j}(t)
$$

where $\alpha_{i j}=\int_{0}^{a} \int_{G} t^{k} u(x, t) \psi_{i}(x) \phi_{j}(t) d x d t$, and the convergence in (4.5) is understood to be in the mean.

Now, define $h_{i}(t)=\int_{G} u(x, t) \psi_{i}(x) d x$. Then $h_{i}(t)$ satisfies the differential equation

$$
\left(t^{k} h_{i}^{\prime}(t)\right)^{\prime}+\lambda_{i} t^{k} h_{i}(t)=0, \quad h_{i}(a)=0
$$

where $\lambda_{i}$ is the eigenvalue of problem (4.4) corresponding to $\psi_{i}(x)$. To see what boundary condition $h_{i}(t)$ satisfies at $t=0$ notice that since $h_{i}(t)$ is the Fourier coefficient of $u(x, t)$, by Parseval's identity we have $\int_{0}^{a} t^{k} h_{i}^{2}(t) d t=\sum_{j=1}^{\infty}\left|\alpha_{i j}\right|^{2}<$ $\infty$. Thus, by Corollary 3.2, equation (4.6) has only the trivial solution if $\lambda_{i}$ is not equal to any of the eigenvalues of

$$
\left(t^{k} h^{\prime}(t)\right)^{\prime}+\lambda t^{k} h(t)=0, \quad \int_{0}^{a} t^{k} h^{2}(t) d t<\infty, \quad h(a)=0 .
$$

However, the eigenvalues of problem (4.7) are the roots to the equation $J_{|1-k| / 2}(\sqrt{\lambda} a)=0$. Thus, equation (4.6) has only the trivial solution when $J_{|1-k| / 2}\left(\sqrt{\lambda_{i} a}\right) \neq 0$. It follows that $\int_{G} u(x, t) \psi_{i}(x) d x \equiv 0$ for $0<t<a$, which implies that $u(x, t) \equiv 0$ since $\left\{\psi_{i}(x)\right\}$ is a complete orthonormal set in $L^{2}(G)$ and $u(x, t)$ is continuous in $G \times(0, a)$.

REMARKS. It can be shown that when $k \leqslant-1$, problem (4.2) is equivalent to

$$
\begin{aligned}
& u_{t t}+\frac{k}{t} u_{t}- \sum_{i, j=1}^{n} D_{i}\left(a_{i j}(x) D_{j} u\right)+c(x) u=0, \\
& \partial u / \partial \nu+\sigma(x) u=0 \quad \text { on } \partial G \times(0, a), \\
& u(x, 0)=u(x, a)=0 \quad \text { for } x \in G .
\end{aligned}
$$

ThEOREM 4.2. Assume $-1<k<3$ and that $u \in C^{2}(\bar{G} \times(0, a))$ is a solution of

$$
\begin{aligned}
& u_{t t}+\frac{k}{t} u_{t}- \sum_{i, j=1}^{n} D_{i}\left(a_{i j}(x) D_{j} u\right)+c(x) u=0 \\
& \partial u / \partial v+\sigma(x) u=0 \quad \text { on } \partial G \times(0, a)
\end{aligned}
$$




$$
\lim _{t \rightarrow 0^{+}} t^{k} W(u(x, \cdot), \bar{\xi} ; t)=0, \quad u(x, a)=0 \text { for } x \in G,
$$

where $W(\cdot, \cdot, t)$ is defined by $(3.5)$ and $\xi\left(t, \lambda_{0}\right)$ is a possibly complex-valued solution of $\left(t^{k} \xi^{\prime}\right)^{\prime}+\lambda_{0} t^{k} \xi=0$ satisfying $\lim _{t \rightarrow 0+} t^{k} W(\xi, \bar{\xi} ; t)=0$. Then $u \equiv 0$ if and only if $l_{n} \neq \lambda_{m}$, where $l_{n}$ and $\lambda_{m}$ are the eigenvalues of the eigenvalue problems (3.6) and (4.4), respectively.

The proof is similar to that of Theorem 4.1 and will be omitted.

REMARKS. The solution

$$
\xi(t)= \begin{cases}-1+A t^{1-k} & \text { for } k \neq 1, \\ -1+A \ln t & \text { for } k=1,\end{cases}
$$

of the equation $\left(t^{k} \xi^{\prime}\right)^{\prime}=0$ satisfies the condition $\lim _{t \rightarrow 0^{+}} t^{k} W(\xi, \bar{\xi} ; t)=0$ if and only if $A$ is real; For this choice of $\xi(t)$, the boundary condition (4.10) at $t=0$ becomes

$$
\begin{array}{ll}
\lim _{t \rightarrow 0^{+}}\left[(1-k) A u(x, t)-\left(A t^{1-k}-1\right) t^{k} u_{t}(x, t)\right]=0 & \text { for } k \neq 1, \\
\lim _{t \rightarrow 0^{+}}\left[A u(x, t)-(A \ln t-1) t u_{t}(x, t)\right]=0 & \text { for } k=1 .
\end{array}
$$

Each different choice of the real constant $A$ yields a different boundary condition for the boundary value problem (4.9)-(4.10). The simplest choice is obtained by choosing $A=0$. Then the boundary condition at $t=0$ that is associated with problem (4.9) is

$$
\lim _{t \rightarrow 0^{+}} t^{k} u_{t}(x, t)=0 \quad \text { for } x \in G .
$$

We shall close by presenting without proof two results that correspond to the Dirichlet and Neumann problems for the class of singular equations under consideration. Notice that these results are valid for all values of $k$, whereas those in [11] are not..

THEOREM 4.3. Let $u \in C^{2}(\bar{G} \times(0, a))$ be a solution of

$$
\begin{aligned}
u_{t t}+\frac{k}{t} u_{t}-\sum_{i, j=1}^{n} D_{i}\left(a_{i j}(x) D_{j} u\right)+c(x) u=0, \\
\partial u / \partial \nu+\sigma(x) u=0 \text { on } \partial G \times(0, a),
\end{aligned}
$$

satisfying

$$
\begin{aligned}
u(x, 0)=u(x, a)=0 & \text { for } x \in G \text { if } k<1, \\
|u(x, 0)|<\infty, \quad u(x, a)=0 & \text { for } x \in G \text { if } 1 \leqslant k .
\end{aligned}
$$


Then $u \equiv 0$ if and only if $J_{|1-k| / 2}(\sqrt{\lambda} a) \neq 0$ where $\lambda_{i}$ are the positive eigenvalues of problem (4.4).

REMARKS. It should be pointed out that Theorem 1 of [11] and Theorem 3.4 are independent even in the case $0<k<1$. Theorem 1 of [11] assumes that $u_{t}(x, t)$ is continuous in $\bar{G} \times[0, a]$, while Theorem 3.4 assumes only that $u_{t}(x, t)$ is continuous in $\bar{G} \times(0, a)$. It is easily shown that Theorem 1 of [11] is false under our less stringent continuity assumptions on $u_{t}(x, t)$. For example, $u(x, t)=t^{(1-k) / 2} J_{|1-k| / 2}(t) \sin x$ is a nontrivial solution of $u_{t t}+(k / t) u_{t}-u_{x x}$ $=0$ satisfying $u(0, t)=u(\Pi, t)=0$, and the initial condition $u(x, 0)=0$. This does not contradict Theorem 1 of [11], however, since while the solution is contained in $C^{2}(\bar{G} \times(0, \Upsilon))$ for any $\Upsilon>0$, it is not contained in $C^{2}(G \times(0, \Upsilon))$ $\cap C^{1}(\bar{G} \times[0, \Upsilon])$ as required by Theorem 1 of [11] because $u_{t}(x, t)=O\left(t^{-k}\right)$.

THEOREM 4.4. Let $u \in C^{2}(\bar{G} \times(0, a))$ be a solution of

$$
\begin{aligned}
u_{t t}+\frac{k}{t} u_{t}-\sum_{i, j=1}^{n} D_{i}\left(a_{i j}(x) D_{j} u\right)+c(x) u=0, \\
\partial u / \partial v+\sigma(x) u=0 \text { on } \partial G \times(0, a), \\
u_{t}(x, 0)=u_{t}(x, a)=0 \quad \text { for } x \in G,
\end{aligned}
$$

and assume that the eigenvalues $\lambda_{i}$ of problem (4.4) are positive. Then $u \equiv 0$ if and only if

$$
J_{(1+k) / 2}\left(\sqrt{\lambda_{i}} a\right) \neq 0 \text {. }
$$

Remarks. Condition (4.13) extends condition (26) of Theorem 3 in [11] to negative values of $k$. It should be pointed out that condition (4.13) is equivalent to

$$
\begin{array}{cc}
Y_{-(1+k) / 2}\left(\sqrt{\lambda_{i}} a\right) \neq 0 & \text { if } k<1, \\
J_{(1+k) / 2}\left(\sqrt{\lambda_{i}} a\right) \neq 0 & \text { if } 1 \leqslant k
\end{array}
$$

where $Y_{p}$ is the Bessel function of the second kind of order $p$.

Acknowledgment. The author would like to thank Professor P. W. Schaefer for some helpful discussions concerning this problem.

\section{REFERENCES}

1. Abdul-Latif and Diaz, Dirichlet, Neumann, and mixed boundary value problems for the wave equation $u_{x x}-u_{y y}=0$ for a rectangle, Applicable Anal. 1 (1974), 1-12.

2. D. G. Bourgin and R. Duffin, The Dirichlet problem for the vibrating string equation, Bull. Amer. Math. Soc. 45 (1939), 851-858. MR 1, 120.

3. J. B. Diaz and E. C. Young, Uniqueness of solutions of certain boundary value problems for ultrahyperbolic equations, Proc. Amer. Math. So,c. 29 (1971), 569-574. MR 44 \#626. 
4. D. R. Dunninger and R. J. Weinacht, Improperly posed problems for singular equations of the fourth order, Applicable Anal. 4 (1975), 331-341.

5. D. R. Dunninger and E. C. Zachmanoglou, The condition for uniqueness of solutions of the Dirichlet problem for the wave equation in coordinate rectangles, J. Math. Anal. Appl. 20 (1967), 17-21. MR 37 \#1807.

6. The condition for uniqueness of the Dirichlet problem for hyperbolic equations in cylindrical domains, J. Math. Mech. 18 (1969), 763-766. MR 38 \#4817.

7. D. W. Fox and C. Pucci, The Dirichlet problem for the wave equation, Ann. Mat. Pura. Appl. (4) 46 (1958), 155-182. MR 21 \#3653.

8. F. John, The Dirichlet problem for a hyperbolic equation, Amer. J. Math. 63 (1941), 141-145. MR 2, 204.

9. M. Picone, Appunti d'analisi superiore, Rondinella, Napoli, 1940, pp. 679-831. MR 3, 144.

10. V. G. Sigillito, $O_{n}$ the uniqueness of solutions of certain improperly posed problems, Proc. Amer. Math. Soc. 24 (1970), 828-831. MR 40 \#7612.

11. E. C. Young, Uniqueness of solutions of the Dirichlet and Neumann problems for hyperbolic equations, Trans. Amer. Math. Soc. 160 (1971), 403-409. MR 43 \#7777.

DEPARTMENT OF MATHEMATICS, UNIVERSITY OF TENNESSEE, KNOXVILLE, TENNESSEE 37916 